\title{
Two-photon decay of heavy quarkonium from heavy-quark spin symmetry*
}

\author{
T. N. Pham \\ Centre de Physique Théorique, CNRS \\ Ecole Polytechnique, 91128 Palaiseau, Cedex, France
}

(Dated: October 28, 2018)

\begin{abstract}
With the recent measurements on $\eta_{c}$ and $\eta_{c}^{\prime}$ at CLEO, Babar and Belle, and with the prospect of finding the $\eta_{b}$ at the Tevatron, it seems appropriate to have another look at the two-photon decay of heavy quarkonium from the standpoint of an effective Lagrangian based on local operator expansion and heavy-quark spin symmetry. In this talk, I would like to discuss a recent work on the two-photon decay rates of ground states and excited states of $\eta_{c}$ and $\eta_{b}$ using the local operator expansion approach and heavy-quark spin symmetry and taking into account the binding-energy. We find that the predicted two-photon width for $\eta_{c}$ agrees well with experiment, but the predicted value for $\eta_{c}(2 S)$ is twice larger than the CLEO estimation. We point out that the essentially model-independent ratio of $\eta_{b}$ two-photon width to the $\Upsilon$ leptonic width and the $\eta_{b}$ two-photon width could be used to extract the strong coupling constant $\alpha_{s}$.
\end{abstract}

PACS numbers: 13.20.Hd,13.25.Gv,11.10.St,12.39Hg

\footnotetext{
* Talk given at the QCD@Work 2007 International Workshop on QCD: Theory and Experiment, Martina Franca, Italy, 16-20 June 2007
} 


\section{INTRODUCTION}

The physics of quarkonium decay seems to be rather well understood within the conventional framework of QCD [1, 2]. However a recent experimental estimation of the two-photon width of the $\eta_{c}^{\prime}$ by the CLEO collaboration [3] gives $\Gamma_{\gamma \gamma}\left(\eta_{c}^{\prime}\right)=1.3 \pm 0.6 \mathrm{keV}$, which contradicts most of the existing theoretical predictions in the range 3.7 to $5.7 \mathrm{keV}$ [4, 5, 6, 7, 8, 9, 10, 11]. This is surprising, since the non-relativistic $\eta_{c}^{\prime} \rightarrow \gamma \gamma$ decay rate differs from that for $\eta_{c}$ only by the wave function at the origin: thus it is difficult to lower the predicted value without considering other effects like binding effects and relativistic corrections. In fact since the first excited state $\eta_{c}^{\prime}$ is more that $600 \mathrm{MeV}$ above the $\eta_{c}$, the mass effects on the decay rate could be important and a better approach would be to use relativistic kinematics in the calculation of the decay rate. In this talk, I would like to discuss a recent work [12] in which we use heavy-quark spin symmetry(HQSS) and an effective Lagrangian from local operator product expansion to relate the two-photon width of charmonium and bottomonium singlet states to the leptonic width of the triplet states. We find that, the predicted two-photon width of $\eta_{c}$ agrees with experiment, but the predicted value for $\eta_{c}^{\prime}$ is twice the CLEO value. For $\eta_{b}, \eta_{b}^{\prime}$ and $\eta_{b}^{\prime \prime}$, the predicted widths are higher than most of the existing calculations.

\section{EFFECTIVE LAGRANGIAN FOR ${ }^{1} S_{0}$ DECAY INTO TWO-PHOTON}

In the two-photon decay of a heavy quarkonium bound state, the outgoing-photon momentum is large compared with the relative momentum of the quark-antiquark bound state, which is $O\left(\Lambda / m_{Q}\right)$, with $\Lambda \ll m_{Q}$. One obtains an effective Lagrangian for the process $c \bar{c} \rightarrow \gamma \gamma$ (represented by the upper diagram in Fig(1) by expanding the heavy quark propagator, e.g. charm quark, in powers of $q^{2} / m_{c}^{2}$, and neglecting $\mathcal{O}\left(q^{2} / m_{c}^{2}\right)$ terms $\left(q=p_{c}-p_{\bar{c}}\right)$. Like leptonic decay, the two-photon decay, in this approximation, is described by the following effective Lagrangian:

$$
\begin{aligned}
& \mathcal{L}_{\text {eff }}^{\gamma \gamma}=-i c_{1}\left(\bar{c} \gamma_{\sigma} \gamma_{5} c\right) \varepsilon_{\mu \nu \rho \sigma} F_{\mu \nu} A_{\rho} \\
& \mathcal{L}_{\text {eff }}^{\ell \bar{\ell}}=-c_{2}\left(\bar{c} \gamma_{\mu} c\right)\left(\ell \gamma_{\mu} \bar{\ell}\right)
\end{aligned}
$$

with

$$
c_{1} \simeq \frac{Q_{c}^{2}\left(4 \pi \alpha_{\mathrm{em}}\right)}{M_{\eta_{c}}^{2}+b_{\eta_{c}} M_{\eta_{c}}}, \quad c_{2}=\frac{Q_{c}\left(4 \pi \alpha_{\mathrm{em}}\right)}{M_{\psi}^{2}} .
$$

The factor $1 /\left(M_{\eta_{c}}^{2}+b_{\eta_{c}} M_{\eta_{c}}\right)$ in $c_{1}$ contains the binding-energy effects (the binding-energy $b$ is defined as $\left.b=2 m_{c}-M\right)$ and is obtained from the denominator of the charm-quark propagator 
$\left(k_{1}, k_{2}\right.$ being the outgoing-photon momenta):

$$
\frac{1}{\left[\left(k_{1}-k_{2}\right)^{2} / 4-m_{c}^{2}\right]} \text {. }
$$

The decay amplitude is then given by the matrix element of the axial vector current $\bar{c} \gamma_{\mu} \gamma_{5} c$ similar to the quarkonium leptonic decay amplitude which is given by the vector current matrix element $\bar{c} \gamma_{\mu} c$ for $J / \psi \rightarrow e^{+} e^{-}$. Thus for decays of $S$-wave quarkonium into two photons or a dilepton pair $\ell \bar{\ell}$, we have:

$$
\begin{aligned}
& \mathcal{M}_{\ell \bar{\ell}}=Q_{c}\left(4 \pi \alpha_{\mathrm{em}}\right) \frac{f_{\psi}}{M_{\psi}} \varepsilon_{\mu}\left(\ell \gamma^{\mu} \bar{\ell}\right) \\
& \mathcal{M}_{\gamma \gamma}=-4 i Q_{c}^{2}\left(4 \pi \alpha_{\mathrm{em}}\right) \frac{f_{\eta_{c}}}{M_{\eta_{c}}^{2}+b_{\eta_{c}} M_{\eta_{c}}} \varepsilon_{\mu \nu \rho \sigma} \varepsilon_{1}^{\mu} \varepsilon_{2}^{\nu} k_{1}^{\rho} k_{2}^{\sigma}
\end{aligned}
$$

where

$$
\left\langle 0\left|\bar{c} \gamma_{\mu} c\right| \psi\right\rangle=f_{\psi} M_{\psi} \varepsilon^{\mu}, \quad\left\langle 0\left|\bar{c} \gamma_{\mu} \gamma_{5} c\right| \eta_{c}\right\rangle=i f_{\eta_{c}} P_{\mu}
$$

from which the decay rates are:

$$
\Gamma_{\ell \bar{\ell}}(\psi)=\frac{4 \pi Q_{c}^{2} \alpha_{\mathrm{em}}^{2} f_{\psi}^{2}}{3 M_{\psi}}, \quad \Gamma_{\gamma \gamma}\left(\eta_{c}\right)=\frac{4 \pi Q_{c}^{4} \alpha_{e m}^{2} f_{\eta_{c}}^{2}}{M_{\eta_{c}}} .
$$

By taking $M_{\psi} f_{\psi}^{2}=12|\psi(0)|^{2}$, we recover the usual non-relativistic expression for the decay rate which, with NLO QCD radiative corrections, are given by

$$
\begin{aligned}
& \Gamma^{N L O}\left({ }^{3} S_{1}\right)=\Gamma^{L O}\left({ }^{3} S_{1}\right)\left(1-\frac{\alpha_{s}}{\pi} \frac{16}{3}\right) \\
& \Gamma^{N L O}\left({ }^{1} S_{0}\right)=\Gamma^{L O}\left({ }^{1} S_{0}\right)\left(1-\frac{\alpha_{s}}{\pi} \frac{\left(20-\pi^{2}\right)}{3}\right) .
\end{aligned}
$$
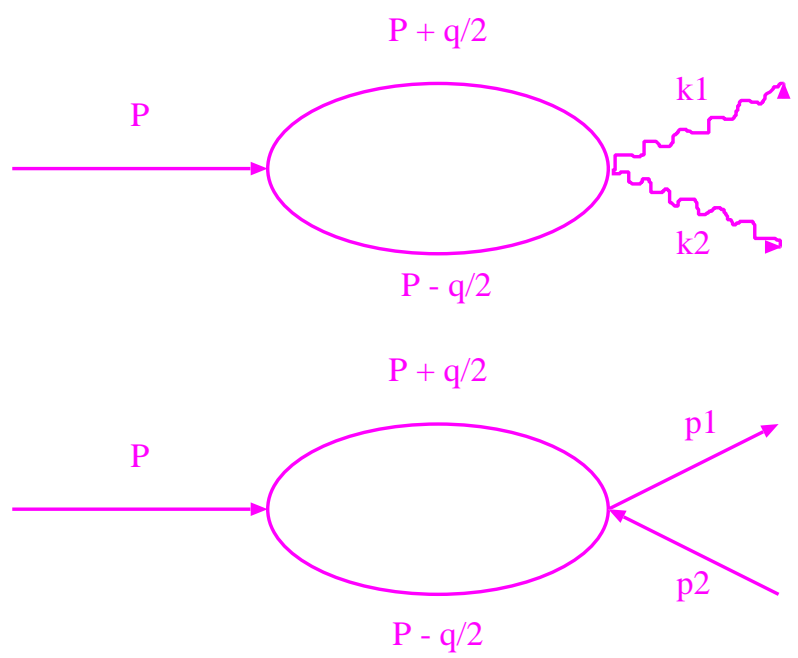

FIG. 1: effective coupling between a $c \bar{c}$ and two-photon (upper) and a lepton pair 


\section{MATRIX ELEMENTS OF LOCAL OPERATORS}

We have shown that in the approximation of neglecting $\mathcal{O}\left(q^{2} / m_{c}^{2}\right)$ terms, the two-photon decay amplitude is given by the $\eta_{c}$ decay constant $f_{\eta_{c}}$. We now derive a symmetry relation between $f_{\eta_{c}}$ and $f_{J / \psi}$, the $J / \psi$ leptonic decay constant using the relativistic spin projection operators for a relativistic Bethe-Salpeter quarkonium bound state.

Consider now the matrix elements of local operators in a fermion-antifermion system with a given spin $S$ and orbital angular momentum $L$ [13, 14] :

$$
\mathcal{A}=\int \frac{d^{4} q}{(2 \pi)^{4}} \operatorname{Tr} \mathcal{O}(0) \chi(P, q)
$$

$P$ is the total 4-momentum of the quarkonium system, $q$ is the relative 4-momentum between the quark and anti-quark and $\chi(P, q)$ is the Bethe-Salpeter wave function. For a quarkonium system in a fixed total, orbital and spin angular momentum, $\chi(P, q)$ is given by ( $\mathbf{q}$ is the relative 3-momentum vector of $q$ ).

$$
\begin{aligned}
\chi\left(P, q ; J, J_{z}, L, S\right) & =\sum_{M, S_{z}} 2 \pi \delta\left(q^{0}-\frac{\mathbf{q}^{2}}{2 m}\right) \psi_{L M}(\mathbf{q})<L M ; S S_{z} \mid J J_{z}> \\
& \times \sqrt{\frac{3}{m}} \sum_{s, \bar{s}} u(P / 2+q, s) \bar{v}(P / 2-q, \bar{s})<\frac{1}{2} s ; \frac{1}{2} \bar{s} \mid S S_{z}> \\
& =\sum_{M, S_{z}} 2 \pi \delta\left(q^{0}-\frac{\mathbf{q}^{2}}{2 m}\right) \psi_{L M}(\mathbf{q}) \mathcal{P}_{S S_{z}}(P, q)<L M ; S S_{z} \mid J J_{z}>.
\end{aligned}
$$

The spin projection operators $\mathcal{P}_{S S_{z}}(P, q)$ are

$$
\begin{aligned}
& \mathcal{P}_{0,0}(P, q)=\sqrt{\frac{3}{8 m^{3}}}[-(P / 2+\not q)+m] \gamma_{5}[(P / 2-\not q)+m] \\
& \mathcal{P}_{1, S_{z}}(P, q)=\sqrt{\frac{3}{8 m^{3}}}[-(P / 2+\not q)+m] \notin\left(S_{z}\right)[(P / 2-\not q)+m] .
\end{aligned}
$$

For $S$-wave quarkonium in a spin singlet $S=0$ and spin triplet $S=1$ state:

$$
\mathcal{A}\left({ }^{2 S+1} S_{J}\right)=\operatorname{Tr}\left(\mathcal{O}(0) \mathcal{P}_{J J_{z}}(P, 0)\right) \int \frac{d^{3} q}{(2 \pi)^{3}} \psi_{00}(q)
$$

In this expression the $q$-dependence in the spin projection operator has been dropped and the integral in Eq.(12) is the $S$-wave function at the origin [14]:

$$
\int \frac{d^{3} q}{(2 \pi)^{3}} \psi_{00}(q)=\frac{1}{\sqrt{4 \pi}} \mathcal{R}_{0}(0) .
$$


Using Eq.(11) and Eq.(12) to compute the matrix elements $\left\langle 0\left|\bar{c} \gamma_{\mu} \gamma_{5} c\right| P\right\rangle$ and $\left\langle 0\left|\bar{c} \gamma_{\mu} c\right| V\right\rangle$ for the singlet $S=0$ pseudo-scalar meson $P$ and for the triplet $S=1$ vector meson $V$, we find, neglecting quadratic $O\left(q^{2}\right)$ terms.

$$
f_{P}=\sqrt{\frac{3}{32 \pi m^{3}}} \mathcal{R}_{0}(0)(4 m), \quad f_{V}=\sqrt{\frac{3}{32 \pi m^{3}}} \mathcal{R}_{0}(0) \frac{\left(M^{2}+4 m^{2}\right)}{M}
$$

$f_{P} / f_{V}$ (from Eq.(14) ) is only quadratic in the binding-energy $b$, and is of the order $O\left(b^{2} / M^{2}\right)$.

Thus the relation $f_{P} \simeq f_{V}$ is valid to a good approximation. It is expected that this relation holds also for excited state of charmonium and bottomonium where the binding terms $O\left(b^{2} / M^{2}\right)$ can be neglected. This is a manifestation of heavy-quark spin symmetry(HQSS). In this limit, the two-photon width of singlet $S=0$ quarkonium state can be obtained from the leptonic width of triplet $S=0$ quarkonium state without using a bound state description. This approach differs from the traditional non-relativistic bound state approach in the use of local operators for which the matrix elements could be measured or extracted from physical quantities, or computed from QCD sum rules [15, 16] and lattice QCD [17].

The ratio of the $\eta_{c}$ two-photon width to $J / \psi$ leptonic width in the limit of HQSS is then:

$$
R_{\eta_{c}}=\frac{\Gamma_{\gamma \gamma}\left(\eta_{c}\right)}{\Gamma_{\ell \bar{\ell}}(J / \psi)}=3 Q_{c}^{2} \frac{M_{J / \psi}}{M_{\eta_{c}}}\left(1+\frac{\alpha_{s}}{\pi} \frac{\left(\pi^{2}-4\right)}{3}\right) .
$$

For $M_{\eta_{c}}=M_{J / \psi}$, the above expression becomes the usual non-relativistic result [18, 19] as mentioned above. From the measured $J / \psi$ leptonic width, we get $\Gamma_{\gamma \gamma}\left(\eta_{c}\right)=7.46 \mathrm{keV}$. Including NLO QCD radiative corrections with $\alpha_{s}=0.26$, we find $\Gamma_{\gamma \gamma}\left(\eta_{c}\right)=9.66 \mathrm{keV}$ in agreement with the world everage value $7.4 \pm 0.9 \pm 2.1 \mathrm{keV}$. A similar result is obtained in [19] who gives $8.16 \pm 0.57 \pm 0.04$ $\mathrm{keV}$.

Thus the effective Lagrangian approach successfully predicts the $\eta_{c}$ two-photon width in a simple, essentially model-independent manner.

\section{HQSS PREDICTIONS FOR $\Gamma_{\gamma \gamma}\left(\eta_{c}^{\prime}\right)$}

To obtain the prediction for $\eta_{c}^{\prime}$, we shall apply HQSS to $2 S$ state. Thus, assuming $f_{\eta_{c}^{\prime}}=f_{\psi^{\prime}}$,

and neglecting binding-energy terms, we find: $\Gamma_{\gamma \gamma}\left(\eta_{c}^{\prime}\right)=\Gamma_{\gamma \gamma}\left(\eta_{c}\right) \frac{f_{\psi^{\prime}}^{2}}{f_{J / \psi}^{2}}=3.45 \mathrm{keV}$. This value is more than twice the evaluation by CLEO, but nearly in agreement with other theoretical calculations [4, 5, 6] as shown in Table 1. Including binding-energy terms, for $M_{\eta_{c}} \simeq M_{J / \psi}, M_{\eta_{c}^{\prime}} \simeq$ $M_{\psi^{\prime}}$, we have

$$
\Gamma_{\gamma \gamma}\left(\eta_{c}^{\prime}\right)=\Gamma_{\gamma \gamma}\left(\eta_{c}\right)\left(\frac{1+b_{\eta_{c}} / M_{\eta_{c}}}{1+b_{\eta_{c}^{\prime}} / M_{\eta_{c}^{\prime}}}\right)^{2} \times\left(\frac{\Gamma_{e^{+} e^{-}}\left(\psi^{\prime}\right)}{\Gamma_{e^{+} e^{-}}(J / \psi)}\right)
$$


which gives

$$
\Gamma_{\gamma \gamma}\left(\eta_{c}^{\prime}\right)=4.1 \mathrm{keV}
$$

\begin{tabular}{|c|c|c|c|c|c|c|c|c|}
\hline$\Gamma_{\gamma \gamma}$ & This work & & {$[5]$} & [6] & [7] & [8] & [9] & [11] \\
\hline$\eta_{c}$ & $7.5-10$ & 4.8 & $7.14 \pm 0$ & $11.8 \pm 0.8 \pm 0.6$ & $3.5 \pm 0.4$ & 5.5 & 5.5 & 6.2 \\
\hline$\eta_{c}^{\prime}$ & $3.5-4.5$ & 3.7 & $4.44 \pm 0.48$ & $5.7 \pm 0.5 \pm 0.6$ & $1.38 \pm 0.3$ & 2.1 & $1.8:$ & $3.36-1.95$ \\
\hline
\end{tabular}

TABLE I: Theoretical predictions for $\Gamma_{\gamma \gamma}\left(\eta_{c}\right)$ and $\Gamma_{\gamma \gamma}\left(\eta_{c}^{\prime}\right)$. (All values are in units of keV).

The measured values are from [20] (PDG) and from CLEO [3] :

$$
\begin{aligned}
& \Gamma_{\gamma \gamma}\left(\eta_{c}\right)=7.4 \pm 0.9 \pm 2.1 \mathrm{keV}, \quad \text { PDG } \\
& \Gamma_{\gamma \gamma}\left(\eta_{c}^{\prime}\right)=1.3 \pm 0.6 \mathrm{keV}, \quad \text { CLEO } .
\end{aligned}
$$

The CLEO extraction of $\Gamma_{\gamma \gamma}\left(\eta_{c}^{\prime}\right)$ is done by considering the following quantity :

$$
R\left(\eta_{c}^{\prime} / \eta_{c}\right)=\frac{\Gamma_{\gamma \gamma}\left(\eta_{c}^{\prime}\right) \times \mathcal{B}\left(\eta_{c}^{\prime} \rightarrow K_{S} K \pi\right)}{\Gamma_{\gamma \gamma}\left(\eta_{c}\right) \times \mathcal{B}\left(\eta_{c} \rightarrow K_{S} K \pi\right)}=0.18 \pm 0.05 \pm 0.02
$$

To obtain $\Gamma_{\gamma \gamma}\left(\eta_{c}^{\prime}\right)$ from the above data, CLEO assumes

$$
\mathcal{B}\left(\eta_{c}^{\prime} \rightarrow K_{S} K \pi\right) \approx \mathcal{B}\left(\eta_{c} \rightarrow K_{S} K \pi\right)
$$

and finds

$$
\Gamma_{\gamma \gamma}\left(\eta_{c}^{\prime}\right)=1.3 \pm 0.6 \mathrm{keV}
$$

On the other hand, Belle measurements of $B \rightarrow \eta_{c} K$ and $B \rightarrow \eta_{c}^{\prime} K$ gives [21]:

$$
R\left(\eta_{c}^{\prime} K / \eta_{c} K\right)=\frac{\mathcal{B}\left(B^{0} \rightarrow \eta_{c}^{\prime} K^{0}\right) \times \mathcal{B}\left(\eta_{c}^{\prime} \rightarrow K_{S} K^{+} \pi^{-}\right)}{\mathcal{B}\left(B^{0} \rightarrow \eta_{c} K^{0}\right) \times \mathcal{B}\left(\eta_{c} \rightarrow K_{S} K^{+} \pi^{-}\right)}=0.38 \pm 0.12 \pm 0.05
$$

Using the approximate equality Eq.(20), one would obtain

$$
\frac{\mathcal{B}\left(B^{0} \rightarrow \eta_{c}^{\prime} K^{0}\right)}{\mathcal{B}\left(B^{0} \rightarrow \eta_{c} K^{0}\right)} \approx 0.4
$$

which agrees more or less with the QCD factorization(QCDF) prediction [23] :

$$
\frac{\mathcal{B}\left(B^{0} \rightarrow \eta_{c}^{\prime} K^{0}\right)}{\mathcal{B}\left(B^{0} \rightarrow \eta_{c} K^{0}\right)} \approx 0.9 \times\left(\frac{f_{\eta_{c}^{\prime}}}{f_{\eta_{c}}}\right)^{2} \approx 0.45
$$

The extracted Belle value Eq.( 22) is close to the ratio obtained from the Babar measured $B^{+} \rightarrow \eta_{c} K^{+}$and $B^{+} \rightarrow \eta_{c}^{\prime} K^{+}$branching ratio[20] .

$$
\frac{\mathcal{B}\left(B^{+} \rightarrow \eta_{c}^{\prime} K^{+}\right)}{\mathcal{B}\left(B^{+} \rightarrow \eta_{c} K^{+}\right)}=0.38 \pm 0.25
$$


This is expected since from $S U(2)$ flavor symmetry, one would have the near equality between the ratios $\mathcal{B}\left(B^{0} \rightarrow \eta_{c}^{\prime} K^{0}\right) / \mathcal{B}\left(B^{0} \rightarrow \eta_{c} K^{0}\right)$ and $\mathcal{B}\left(B^{+} \rightarrow \eta_{c}^{\prime} K^{+}\right) / \mathcal{B}\left(B^{+} \rightarrow \eta_{c} K^{+}\right)$.

Thus the assumption of the approximate equality between the $\eta_{c}^{\prime} \rightarrow K K \pi$ and $\eta_{c} \rightarrow K K \pi$ branching ratio seems to be justified to some extent. This implies the small $\eta_{c}^{\prime} \rightarrow \gamma \gamma$ decay rate quoted above. We note however that the good agreement with QCDF predictions for the measured ratio $\mathcal{B}\left(B^{0} \rightarrow \eta_{c}^{\prime} K^{0}\right) / \mathcal{B}\left(B^{0} \rightarrow \eta_{c} K^{0}\right)$ and $\mathcal{B}\left(B^{+} \rightarrow \eta_{c}^{\prime} K^{+}\right) / \mathcal{B}\left(B^{+} \rightarrow \eta_{c} K^{+}\right)$at Belle and Babar suggests that $f_{\eta_{c}^{\prime}} / f_{\eta_{c}} \approx f_{\psi^{\prime}} / f_{J / \psi}$, which in turn supports our predicted value for the $\eta_{c}^{\prime}$ two-photon width which is more than twice bigger than the CLEO estimated value shown above. More precisely, comparing $R\left(\eta_{c}^{\prime} / \eta_{c}\right)$ with $R\left(\eta_{c}^{\prime} K / \eta_{c} K\right)$ and using QCDF value given in Eq.(24), we find

$$
R\left(\eta_{c}^{\prime} / \eta_{c}\right) \approx R\left(\eta_{c}^{\prime} K / \eta_{c} K\right) / 0.9
$$

The Belle data in Eq.(22) would then implies $R\left(\eta_{c}^{\prime} / \eta_{c}\right) \approx 0.42 \pm 0.13 \pm 0.05$, twice bigger than the CLEO data shown in Eq.(19).

\section{HQSS PREDICTIONS FOR $\Gamma_{\gamma \gamma}\left(\eta_{b}\right)$ AND $\Gamma_{\gamma \gamma}\left(\eta_{b}^{\prime}\right)$}

Since the $b$-quark mass is significantly higher than the $c$-quark mass, the effective Lagrangian and HQSS approach should work better for bottomonia decays to leptons and photons. We thus have:

$$
R_{\eta_{b}}=\frac{\Gamma_{\gamma \gamma}\left(\eta_{b}\right)}{\Gamma_{\ell \bar{\ell}}(\Upsilon)}=3 Q_{b}^{2} \frac{M_{\Upsilon}}{M_{\eta_{b}}}\left(1+\frac{\alpha_{s}}{\pi} \frac{\left(\pi^{2}-4\right)}{3}\right)
$$

(neglecting the small $b_{\eta_{b}} / M_{\eta_{b}}$ binding-energy term). This gives $\Gamma_{\gamma \gamma}\left(\eta_{b}\right)=560 \mathrm{eV}\left(\alpha_{s}\left(M_{\Upsilon}\right)=0.16\right.$, $\left.M_{\eta_{b}}=9300 \mathrm{MeV}\right)$.

For $\eta_{b}^{\prime}$ and higher excited state, one has $\left(M_{\eta_{b}} \simeq M_{\Upsilon}\right.$ and $\left.M_{\eta_{b}^{\prime}} \simeq M_{\Upsilon^{\prime}}\right)$ :

$$
\Gamma_{\gamma \gamma}\left(\eta_{b}^{\prime}\right)=\Gamma_{\gamma \gamma}\left(\eta_{b}\right)\left(\frac{1+b_{\eta_{b}} / M_{\eta_{b}}}{1+b_{\eta_{b}^{\prime}} / M_{\eta_{b}^{\prime}}}\right)^{2}\left(\frac{\Gamma_{e^{+} e^{-}}\left(\Upsilon^{\prime}\right)}{\Gamma_{e^{+} e^{-}}(\Upsilon)}\right) .
$$

which gives $\Gamma_{\gamma \gamma}\left(\eta_{b}^{\prime}\right)=250 \mathrm{eV}$ and $\Gamma_{\gamma \gamma}\left(\eta_{b}^{\prime \prime}\right)=187 \mathrm{eV}$. In Table. II we give our prediction for the two-photon width of $\eta_{b}, \eta_{b}^{\prime}$ and $\eta_{b}^{\prime \prime}$ together with other theoretical predictions. We note that our predicted values are somewhat higher than these predicted values.

Eq.(27) can be used to determine in a reliable way the value of $\alpha_{s}$. The momentum scale at which $\alpha_{s}$ is to be evaluated here could be in principle be fixed with $R_{\eta_{b}}$. 
Further check of consistency of the value for $\alpha_{s}$ may be possible in future mesurements on the $\eta_{b}$ and its two-photon decay branching ratio:

$$
\frac{\Gamma_{\gamma \gamma}\left(\eta_{b}\right)}{\Gamma_{g g}\left(\eta_{b}\right)}=\frac{9}{2} Q_{b}^{4} \frac{\alpha_{e m}^{2}}{\alpha_{s}^{2}}\left(1-7.8 \frac{\alpha_{s}}{\pi}\right)
$$

\begin{tabular}{cccccccccccc}
\hline$\Gamma_{\gamma \gamma}$ & This work & $24]$ & {$[25]$} & {$[4]$} & {$[5]$} & {$[6]$} & {$[\underline{7}]$} & $\underline{[9]}$ & {$[26]$} & {$[27]$} & {$[28]$} \\
\hline$\eta_{b}$ & 560 & 460 & 230 & 170 & $384 \pm 47$ & 520 & $220 \pm 40$ & 350 & 214 & $466 \pm 101$ & $659 \pm 92$ \\
$\eta_{b}^{\prime}$ & 269 & 200 & 70 & - & $191 \pm 25$ & - & $110 \pm 20$ & 150 & 121 & - & - \\
$\eta_{b}^{\prime \prime}$ & 208 & - & 40 & - & - & - & $84 \pm 12$ & 100 & 90.6 & - & - \\
\hline
\end{tabular}

TABLE II: Summary of theoretical predictions for $\Gamma_{\gamma \gamma}\left(\eta_{b}\right), \Gamma_{\gamma \gamma}\left(\eta_{b}^{\prime}\right)$ and $\Gamma_{\gamma \gamma}\left(\eta_{b}^{\prime \prime}\right)$. (All values are in units of $\mathrm{eV})$.

\section{CONCLUSION}

We have shown in this work that effective Lagrangian approach and HQSS can be used to compute quarkonium decays into lepton and photon with relativistic kinematic, for both ground state and excited state of heavy quarkonium systems. Our predicted $\eta_{c}^{\prime} \rightarrow \gamma \gamma$ width is larger than the CLEO estimated value, though many relativistic calculations could give a smaller value for $\eta_{c}^{\prime} \rightarrow \gamma \gamma$ width but also produce smaller value for the $\eta_{c} \rightarrow \gamma \gamma$ width.

Measurements of the two-photon widths for $\eta_{b}$ and higher excited states could provide a test for HQSS and a determination of the $\alpha_{s}$ coupling constant at the scale around the $\Upsilon$ mass, as has been done with the $\Upsilon$ leptonic width in the past.

\section{ACKNOWLEDGMENTS}

I would like to thank G. Nardulli, P. Colangelo, F. De Fazio and the organizers for the warm hospitality extended to me at Martina Franca.

[1] N. Brambilla et al., HEAVY QUARKONIUM PHYSICS, CERN Yellow Report, CERN-2005-005, 2005 Geneva : CERN, 487 pp arXiv:hep-ph/0412158.

[2] For a recent review on open and hidden charm spectroscopy, see, e.g. P. Colangelo, F. De Fazio, R. Ferrandes, and S. Nicotri, Talk given at the Workshop "Continuous Advances in QCD 2006", Minneapolis, USA, May 11-14, 2006, hep-ph/0609240 and other references quoted therein. 
[3] D. M. Asner et al. [CLEO Collaboration], Phys. Rev. Lett. 92, 142001 (2004).

[4] E. S. Ackleh and T. Barnes, Phys. Rev. D 45, 232 (1992).

[5] C. S. Kim, T. Lee and G. L. Wang, Phys. Lett. B 606, 323 (2005).

[6] M. R. Ahmady and R. R. Mendel, Phys. Rev. D 51, 141 (1995).

[7] C. R. Münz, Nucl. Phys. A 609, 364 (1996).

[8] K. T. Chao, H. W. Huang, J. H. Liu and J. Tang, Phys. Rev. D 56, 368 (1997).

[9] D. Ebert, R. N. Faustov and V. O. Galkin, Mod. Phys. Lett. A 18, 601 (2003).

[10] S. B. Gerasimov and M. Majewski, arXiv:hep-ph/0504067.

[11] H. W. Crater, C. Y. Wong and P. Van Alstine, Phys. Rev. D 74, 054028 (2006).

[12] J. P. Lansberg and T. N. Pham, Phys. Rev. D 74, 034001 (2006); ibid D 75, 017501 (2007).

[13] J. H. Kühn, J. Kaplan and E. G. O. Safiani, Nucl. Phys. B 157, 125 (1979).

[14] B. Guberina, J. H. Kühn, R. D. Peccei, and R. Ruckl, Nucl. Phys. B 174, 317 (1980).

[15] V. A. Novikov, L. B. Okun, M. A. Shifman, A. I. Vainshtein, M. B. Voloshin and V. I. Zakharov, Phys. Rept. 41, 1 (1978).

[16] L. J. Reinders, H. R. Rubinstein and S. Yazaki, Phys. Lett. B 113, 411 (1982).

[17] J. J. Dudek, R. G. Edwards and D. G. Richards, Phys. Rev. D 73, 074507 (2006).

[18] W. Kwong, P. B. Mackenzie, R. Rosenfeld and J. L. Rosner, Phys. Rev. D 37, 3210 (1988).

[19] N. Fabiano and G. Pancheri, Eur. Phys. J. C 22, 421 (2002).

[20] Particle Data Group, Review of Particle Physics, J. Phys. G:Nucl. Part. Phys. 32, 1 (2006).

[21] S. K. Choi et al. [BELLE collaboration], Phys. Rev. Lett. 89, 102001 (2002) [Erratum-ibid. 89, 102001 (2002)].

[22] After the Martina Franca Workshop, we learned that H. Nakazawa at the Photon2007 International Conference, Paris 9-13 July (2007), reported Belle latest results on the two-photon width as: $7.48 \pm 0.20$ $\mathrm{keV}$ for $\eta_{c}(1 S)$ and $0.59 \pm 0.13 \mathrm{keV}$ for $\eta_{c}(2 S)$, see H.Nakazawa, talk at the Paris Photon2007 conference.

[23] Z. z. Song, C. Meng and K. T. Chao, Eur. Phys. J. C 36, 365 (2004).

[24] G. A. Schuler, F. A. Berends and R. van Gulik, Nucl. Phys. B 523, 423 (1998).

[25] O. Lakhina and E. S. Swanson, Phys. Rev. D 74 (2006) 014012.

[26] S. Godfrey and N. Isgur, Phys. Rev. D 32, 189 (1985).

[27] N. Fabiano, Eur. Phys. J. C 26, 441 (2003).

[28] A. A. Penin, A. Pineda, V. A. Smirnov and M. Steinhauser, Nucl. Phys. B 699, 183 (2004). . 